\title{
Dominio del idioma inglés en estudiantes de nuevo ingreso universitario: información para la toma de decisiones
}

English language knowledge dominion on first year university students: Information for decision making

Proficiência na língua inglesa em novos estudantes universitários: informações para a tomada de decisão

Ángel Esteban Torres-Zapata

Universidad del Valle de México, Campus Villahermosa, México macronutriente@hotmail.com https://orcid.org/0000-0002-6066-3258

Teresa del Jesús Brito-Cruz Universidad Autónoma del Carmen, México tbrito@pampano.unacar.mx https://orcid.org/0000-0002-7030-4187

José Rafael Villanueva-Echavarría Universidad Autónoma del Carmen, México jvillanueva@pampano.unacar.mx https://orcid.org/0000-0001-6227-4793 


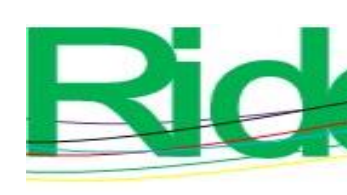

Revista Iberoamericana para la
Investigación y el Desarrollo Educativo
ISSN $2007-7467$

\section{Resumen}

El dominio de una lengua extranjera, específicamente el idioma inglés en América Latina, es una necesidad para todo profesional debido al proceso de globalización. Este fenómeno ha sido el principal motivo por el cual dicha lengua ha adquirido importancia a nivel mundial. Por tanto, el objetivo de la presente investigación fue caracterizar el dominio de conocimientos del idioma inglés en estudiantes de nuevo ingreso de la Facultad de Ciencias de la Salud de una universidad pública en el sureste de México, lo cual permitirá la toma de decisiones en cuanto a la actualización del currículo de los programas educativos. Para ello, se desarrolló un estudio con enfoque cuantitativo; la muestra fue de tipo no probabilístico, y se consideró a la población total de estudiantes de nuevo ingreso de la facultad (generación 2020). El instrumento para la recolección de los datos fue diseñado por el cuerpo académico adjunto al centro de idiomas de la institución y organizado en tres secciones I: Auditiva; II: Lectura, y III: Uso del vocabulario y gramática. La evaluación diagnóstica del idioma inglés versión en línea se llevó a cabo en el mes de julio de 2020 y el análisis de datos se realizó durante el mes de agosto de 2020. Los resultados fueron procesados con el paquete estadístico SPSS, versión 25.0, para Windows. Se realizó un análisis descriptivo. El estudio estuvo conformado por una población de 602 estudiantes de nuevo ingreso de seis programas educativos de la facultad, de los cuales $n=424(70.43 \%)$ corresponden al género femenino y n $=178$ (29.57\%) al masculino. Los resultados demuestran que $77.41 \%$ se encuentra en un nivel preliminar Básico. De hecho, el programa donde se evidencia el mejor desempeño es Psicología, mientras que el más bajo se encuentra en Educación Física. En síntesis, los niveles de logro alcanzados por los estudiantes están por debajo de la media nacional, lo que significa que aún requieren más tiempo de capacitación. Además, se deben identificar las deficiencias y dificultades en el aprendizaje del idioma inglés en los niveles académicos anteriores. Por ello, es posible concluir que la universidad debe actualizar su currículo y sus orientaciones pedagógicas para mejorar el dominio del idioma inglés y con ello la calidad de la educación profesional.

Palabras clave: dominio del conocimiento, estudiantes universitarios, idioma inglés. 


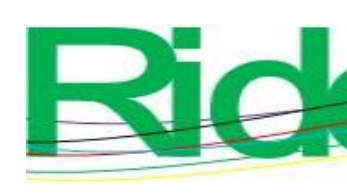

Revista Iberoamericana para la Investigación y el Desarrollo Educativo ISSN $2007-7467$

\section{Resumo}

O domínio de uma língua estrangeira, especificamente a língua inglesa na América Latina, é uma necessidade para todo profissional devido ao processo de globalização. Esse fenômeno tem sido o principal motivo pelo qual esse idioma adquiriu importância mundial. Portanto, o objetivo desta pesquisa foi caracterizar o domínio da língua inglesa em novos alunos da Faculdade de Ciências da Saúde de uma universidade pública do sudeste do México, o que permitirá a tomada de decisão quanto à atualização do currículo dos programas educacionais. Para isso, foi desenvolvido um estudo com abordagem quantitativa; A amostra foi não probabilística, e foi considerada a população total de novos alunos do corpo docente (geração 2020). O instrumento de coleta de dados foi elaborado pelo corpo acadêmico vinculado ao Centro de Línguas da instituição e organizado em três seções I: Audição; II: Leitura e III: Uso de vocabulário e gramática. A versão online da avaliação diagnóstica da língua inglesa foi realizada em julho de 2020 e a análise dos dados realizada em agosto de 2020 . Os resultados foram processados com o pacote estatístico SPSS, versão 25.0, para Windows. Foi feita uma análise descritiva. $\mathrm{O}$ estudo constituiu-se de uma população de 602 novos alunos de seis programas de ensino da faculdade, dos quais $n=424(70,43 \%)$ correspondem ao gênero feminino en $=178(29,57 \%)$ ao gênero masculino. Os resultados mostram que 77,41\% estão no nível básico preliminar. Na verdade, o programa onde se evidencia o melhor desempenho é a Psicologia, enquanto o pior é a Educação Física. Em suma, os níveis de aproveitamento alcançados pelos alunos estão abaixo da média nacional, o que significa que ainda requerem mais tempo de formação. Além disso, devem ser identificadas deficiências e dificuldades no aprendizado da língua inglesa em níveis acadêmicos anteriores. Portanto, é possível concluir que a universidade deve atualizar seu currículo e suas diretrizes pedagógicas para aprimorar o domínio da língua inglesa e com ela a qualidade da formação profissional.

Palavras-chave: domínio do conhecimento, estudantes universitários, língua inglesa. Fecha Recepción: Noviembre 2020 Fecha Aceptación: Mayo 2021 


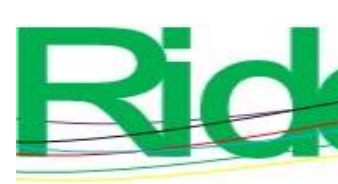

Revista Iberoamericana para la
Investigación y el Desarrollo Educativo
ISSN $2007-7467$

complemento necesario para promover la formación integral y la calidad educativa en un mundo globalizado (Carranza, Islas y Maciel, 2018; España, 2010).

Ahora bien, el aprendizaje de una segunda lengua en un entorno académico es un proceso que se planifica y ejecuta en un marco institucional con el fin de desarrollar la competencia lingüística de los estudiantes (Oñate, 2016). Este aprendizaje propone que la metabolización de dicho idioma se fundamente en los mismos principios que rigen el dominio de la lengua materna, incluso cuando la gramática completa del segundo idioma no se consigue mediante factores no lingüísticos (Tapia, 2018; Rueda y Wilburn, 2014). La diferencia está en que el primer idioma se adquiere a través de la inmersión en un ambiente nativo, mientras que la lengua extranjera se desarrolla en un ambiente controlado en el aula, donde comúnmente los errores se producen en aquellos puntos donde la lengua materna y la extranjera son distintas (Oñate, 2016). El objetivo es que los estudiantes consigan competencias equivalentes a los estándares internacionales para estar preparados frente a los cambios futuros de los sistemas de educación y de empleabilidad (León-Jiménez et al., 2018).

Para evaluar de manera adecuada el desarrollo de esta competencia en los aspirantes universitarios de nuevo ingreso es necesario aplicarles una prueba diagnóstica no para condicionar su admisión, sino para determinar en cuál nivel de aprendizaje del idioma inglés deberá iniciar en la universidad. Este proceso exploratorio resulta esencial porque les permite a los organismos reguladores valorar la trascendencia de un programa educativo en cuanto a la inserción laboral de sus egresados (Universidad Autónoma del Carmen [Unacar], 2017).

Por este motivo, el objetivo de la presente investigación fue describir el dominio de conocimientos en el idioma inglés de estudiantes de nuevo ingreso de la Facultad de Ciencias de la Salud de una universidad pública en el sureste de México con el fin de emprender acciones que permitan actualizar el currículo de los programas educativos de dicha facultad.

En esta institución se ha identificado que el nivel más alto de rezago educativo se presenta en los cursos que contribuyen a las competencias genéricas, especialmente en comunicación y relación social (CRS), donde se aborda la habilidad para comunicarse y relacionarse tanto en español como en una segunda lengua. Esa competencia genérica se incluye en todos los programas educativos de la institución y está formada por cuatro niveles curriculares y dos cursos remediales obligatorios no curriculares. 


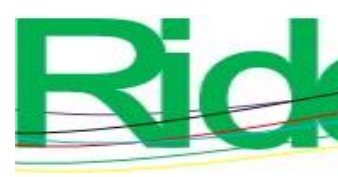

Revista Iberoamericana para la Investigación y el Desarrollo Educativo ISSN 2007-7467

\section{Materiales y métodos}

El enfoque de la presente investigación fue cuantitativo, con un alcance exploratorio, descriptivo y un diseño experimental transversal. La población de estudio fue seleccionada de manera no probabilística, para lo cual se incluyó a la totalidad de alumnos de primer ciclo de la generación 2020 de los programas educativos de la Facultad de Ciencias de la Salud de una universidad mexicana. La evaluación diagnóstica del idioma inglés, versión en línea, se llevó a cabo del 6 al 10 de julio de 2020, y el análisis de datos se realizó durante el mes de agosto de 2020 .

Se excluyeron los casos de alumnos que no sustentaron la evaluación en línea en tiempo y forma del periodo antes mencionado, aun cuando fueran aceptados de manera posterior en los programas educativos.

La consulta de la base de datos que se utilizó fue proporcionada por la coordinación del centro de idiomas de la institución, unidad administrativa responsable de generar, resguardar y custodiar la información de los resultados alcanzados de cada sustentante.

El instrumento empleado fue diseñado por el cuerpo académico adjunto al centro de idiomas de la institución, y fue aplicado en línea debido a la situación generada por la pandemia. En concreto, el instrumento quedó conformado por tres secciones:

- Sección I: Comprensión auditiva. Quedó constituida por 20 reactivos, cada uno con cuatro opciones de respuestas

- Sección II: Lectura. Estuvo conformada por 20 reactivos, cada uno con cuatro opciones de respuesta.

- Sección III: Uso vocabulario y gramática. Esta fue elaborada con 120 reactivos, cada uno con cuatro opciones de respuesta. Este último apartado quedó dividido en seis bloques de 20 reactivos cada uno. El objetivo fue evaluar los conocimientos de cada nivel impartido, ya sea de manera curricular obligatoria o como contenido de los cursos remediales no curriculares. El primer bloque de reactivos (1 al 20) se enfocó en los contenidos del curso remedial Nivelación A; el segundo bloque (del 21 al 40) en los contenidos del curso remedial Nivelación B, y así sucesivamente.

En la tabla 1 se indican los contenidos valorados en cada bloque dentro de la institución en estudio. 
Tabla 1. Correlación entre los reactivos del examen diagnóstico

\begin{tabular}{|r|c|c|c|c|c|c|}
\hline Bloque & I & II & III & IV & V & VI \\
\hline Reactivo del ... al ... & $1-20$ & $21-40$ & $41-60$ & $61-80$ & $81-100$ & $101-120$ \\
\hline $\begin{array}{r}\text { Curso que representa } \\
\text { en la institución }\end{array}$ & Nivelación A & Nivelación B & $\begin{array}{c}\text { Inglés } \\
\text { I }\end{array}$ & $\begin{array}{c}\text { Inglés } \\
\text { II }\end{array}$ & Inglés III & Inglés IV \\
\hline
\end{tabular}

Fuente: Unacar (2017)

Los reactivos del instrumento pertenecen a los contenidos de los diferentes cursos de inglés general que ofrece la institución de estudio. Esto permite obtener una referencia diagnóstica de cada uno de los aspirantes de ingreso y del contenido que deben acreditar durante su tránsito por la institución.

Este instrumento tiene dos objetivos definidos: primero, medir el conocimiento del idioma inglés al momento de ingresar a la universidad. Esta información permite comprar el nivel de conocimiento del estudiante cuando ingresó a la universidad con el nivel de conocimiento al finalizar sus cursos de inglés. Es necesario mencionar que esta evaluación diagnóstica no juega papel alguno en la admisión de la universidad. Segundo, en función del puntaje obtenido, el estudiante tiene la oportunidad de acreditar los cursos de nivelación A y nivelación B, así como el derecho de presentar los exámenes de acreditación de los siguientes cuatro niveles, según logre obtener el puntaje mínimo satisfactorio para cada nivel (tabla 2).

Tabla 2. Puntaje mínimo para acreditación de niveles de inglés

\begin{tabular}{|r|c|c|c|c|c|c|}
\hline Bloque & I & II & III & IV & V & VI \\
\hline Reactivo del ... al ... & $1-20$ & $21-40$ & $\begin{array}{c}41- \\
60\end{array}$ & $61-80$ & $81-100$ & $101-120$ \\
\hline $\begin{array}{r}\text { Número mínimo de reactivos correctos } \\
\text { en cada bloque para acreditación }\end{array}$ & 16 & 16 & 14 & 14 & 14 & 14 \\
\hline
\end{tabular}

Fuente: Unacar (2017)

En lo referente a la sección auditiva y de lectura, en caso de que el estudiante haya obtenido 15 aciertos en alguno de los dos primeros grupos de 20 reactivos gramaticales, y 13 en el caso de los siguientes cuatro grupos de 20 reactivos gramaticales, se determina que el estudiante se encuentra apto para el siguiente nivel.

Como se observa en la tabla 2 , el número de aciertos requeridos para la acreditación de los cursos de nivelación A y B es mayor que el puntaje requerido en los cuatro niveles posteriores para ser canalizados a exámenes de acreditación. Lo anterior tiene como propósito garantizar que el estudiante cuente con los conocimientos mínimos necesarios en estos dos niveles básicos del idioma inglés, dado que estos niveles no transitan por exámenes de acreditación, y es el instrumento diagnóstico el método empleado para acreditar estas 


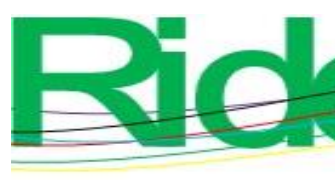

Revista Iberoamericana para la Investigación y el Desarrollo Educativo ISSN 2007 - 7467

Tabla 4. Distribución del nivel de dominio del idioma inglés

\begin{tabular}{|c|c|c|r|r|}
\hline Universidad & MCER & CENNI & $\mathrm{n}$ & $\%$ \\
\hline Nivelación A & A1 & Básico 1 & 399 & 66.28 \\
\hline Nivelación B & A1+ & Básico 2 & 67 & 11.13 \\
\hline Inglés I & A2- & Pre-intermedio 1 & 64 & 10.63 \\
\hline Presentar examen de acreditación de Inglés I-4 & 72 & 11.96 \\
\hline \multicolumn{2}{|l|}{ Total } & 602 & 100 \\
\hline
\end{tabular}

Fuente: Elaboración propia

En la tabla 5 se aprecia el nivel de dominio del idioma inglés por programa educativo en la facultad. En tal sentido, se puede indicar que en Psicología casi $60 \%$ de los estudiantes de nuevo ingreso tienen un dominio del idioma inglés en el nivel Básico, aunque dicha situación tiene mayor prevalencia en los demás programas educativos; una muestra son Enfermería y Educación Física y Deportes, donde se presentan más casos en ese nivel.

Tabla 5. Nivel de dominio del idioma inglés por programa educativo

\begin{tabular}{|c|c|c|c|c|c|c|c|c|}
\hline \multirow{3}{*}{ Programa educativo } & \multicolumn{4}{|c|}{ Básico } & \multirow{2}{*}{\multicolumn{2}{|c|}{\begin{tabular}{|c}
$\begin{array}{c}\text { Pre- } \\
\text { intermedio }\end{array}$ \\
Inglés I
\end{tabular}}} & \multirow{2}{*}{\multicolumn{2}{|c|}{$\begin{array}{l}\text { Presentar } \\
\text { examen } \\
\text { de } \\
\text { acreditaci } \\
\text { ón }\end{array}$}} \\
\hline & \multicolumn{2}{|c|}{$\begin{array}{c}\text { Nivelación } \\
\text { A }\end{array}$} & \multicolumn{2}{|c|}{$\begin{array}{c}\text { Nivelació } \\
\text { n B }\end{array}$} & & & & \\
\hline & $\mathrm{f}$ & $\%$ & $\mathrm{f}$ & $\%$ & $\mathrm{f}$ & $\%$ & $\mathrm{f}$ & $\%$ \\
\hline $\begin{array}{l}\text { Licenciatura en Educación Física y Deportes } \\
\text { (LEFYD) }\end{array}$ & 20 & 86.96 & 2 & 8.70 & 1 & 4.35 & 0 & 0.00 \\
\hline Licenciatura en Enfermería (LE) & 80 & 80.81 & 11 & 11.11 & 4 & 4.04 & 4 & 4.04 \\
\hline Licenciatura en Fisioterapia (LF) & 47 & 65.28 & 8 & 11.11 & 11 & 15.28 & 6 & 8.33 \\
\hline Licenciatura en Medicina (LM) & $\begin{array}{l}17 \\
0\end{array}$ & 60.50 & 33 & 11.74 & 32 & 11.39 & 46 & 16.37 \\
\hline Licenciatura en Nutrición (LN) & 40 & 74.07 & 5 & 9.26 & 5 & 9.26 & 4 & 7.41 \\
\hline Licenciatura en Psicología (LP) & 42 & 57.53 & 8 & 10.96 & 11 & 15.07 & 12 & 16.44 \\
\hline
\end{tabular}

Fuente: Elaboración propia

En la tabla 6 se visualiza el puntaje obtenido por cada sección del instrumento diagnóstico y por programa educativo de la institución de estudio. 


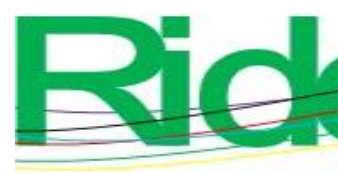

Revista Iberoamericana para la Investigación y el Desarrollo Educativo ISSN $2007-7467$

En concreto, los resultados del examen diagnóstico de inglés aplicado indican que, en promedio, $77.41 \%$ de estudiantes de nuevo ingreso de la facultad se encuentran dentro del nivel preliminar (Nivelación A y B), lo que significa que necesitan más tiempo de capacitación.

Estos resultados son relativamente superiores a los reportados por León-Jiménez et al. (2018), quienes expusieron los siguientes porcentajes en estas facultades: medicina (61.1 $\%)$, ingeniería (60.0\%), derecho $(64.9 \%)$, humanidades $(81.8 \%)$ y ciencias empresariales (81.4\%), mientras que los porcentajes conseguidos por Ibáñez (2008) son más similares a los detallados en la presente investigación, pues dicho autor encontró un nivel preliminar de $70 \%$ de los estudiantes de nuevo ingreso.

Por otra parte, al comparar los resultados entre los programas educativos de la institución de estudio, se observa que el siguiente orden en una escala de mayor dominio a menor: 1) licenciatura en Psicología, 2) licenciatura en Fisioterapia, 3) licenciatura en Medicina, 4) licenciatura en Nutrición, 5) licenciatura en Enfermería, y 6) licenciatura en Educación Física y Deporte.

Este bajo dominio general de la lengua inglesa exige de la universidad una mayor inversión económica, ya que para homologar a los estudiantes en dicho idioma la institución debe ofertar una mayor cantidad de cursos, lo que se traduce en más aulas y docentes.

Sin embargo, también se debe indicar que en el presente trabajo ciertas variables no controladas podrían haber alterado en alguna medida los datos recabados, como la falta de tiempo propuesto para resolver la prueba, la actual situación sanitaria generada por la covid19, el poco interés acerca del tema por parte de los estudiantes y la falta de estudios previos o de práctica (León-Jiménez et al., 2018). Asimismo, se debe considerar que el diagnóstico se desarrolló en línea, lo cual pudo haber exigido de los alumnos conocimientos con los que no estaban familiarizados para emplear la plataforma, así como problemas de conexión, etc.

Aun así, desde el primer ciclo de todas las carreras se debe trabajar en la enseñanza de ese idioma, lo cual se puede potenciar de dos maneras: la primera, contando con docentes cuya lengua materna sea el inglés, ya que de ese modo se puede optimizar todo lo referido a vocabulario, escritura, lectura, gramática y diálogo. La segunda, ofreciendo a los profesores cuya lengua materna no sea el inglés metodologías que despierten el interés en los estudiantes (Buitrago y Herrera, 2016). 


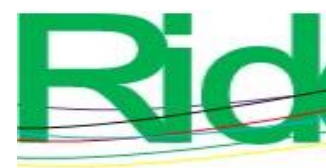

Revista Iberoamericana para la
Investigación y el Desarrollo Educativo
ISSN $2007-7467$

\section{Conclusiones}

Los resultados alcanzados por los estudiantes de nuevo ingreso en la prueba diagnóstica de dominio del idioma inglés demuestran que dichos alumnos se hallan debajo de los estándares requeridos por la Secretaría de Educación Pública de México. Este fenómeno es preocupante si se considera que los estudiantes deben consultar documentos académicos en inglés como parte del material de referencia dentro de las unidades de aprendizaje durante los cuatro años que contemplan los programas de estudio.

Por eso, la universidad debe procurar actualizar el currículo y la orientación pedagógica para promover el desarrollo de las competencias de comunicación que permitan el dominio del idioma inglés. De hecho, más allá de una inclusión curricular, deberá existir pertinencia y vinculación interdisciplinaria del inglés como parte esencial de la integralidad del conocimiento que impone la mundialización, pues así se pueden enfrentar los embates de la globalización y de la era de la información para lograr el desarrollo requerido.

\section{Futuras líneas de investigación}

Se debe identificar el origen de las dificultades que tienen los estudiantes universitarios para dominar el idioma inglés como segunda lengua. Asimismo, falta profundizar en la percepción de los alumnos y de los profesores en cuanto a la enseñanza de ese idioma, así como en el impacto del currículo y de los factores que inciden en su dominio. 


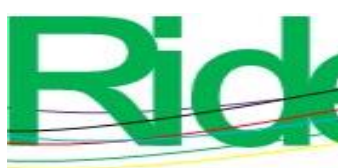

Revista Iberoamericana para la
Investigación y el Desarrollo Educativo
ISSN $2007-7467$

\section{Referencias}

Buitrago, R. y Herrera, R. (2016). Las representaciones gráficas del conocimiento como técnicas de aprendizaje acelerado sugestivo en el aprendizaje del inglés como lengua extranjera. Reflexiones, 6, 67-73. Doi: doi:10.18041/20115768/reflexiones.0.2016.4349

Burgos, B. y López, K. (2010). La situación del mercado laboral de profesionistas. Revista de la Educación Superior, 39(156), 19-33. Recuperado de http://wwmw.scielo.org.mx/scielo.php?script=sci_arttext\&pid=S0185$27602010000400002 \& \operatorname{lng}=\mathrm{es} \& \operatorname{tlng}=\mathrm{es}$

Carranza, M., Islas, C. y Maciel, M. (2018). Percepción de los estudiantes respecto del uso de las TIC y el aprendizaje del idioma inglés. Apertura, 10(2), 50-63. Doi: doi.org/10.32870/ap.v10n2.1391

Chávez-Zambano, M., Saltos-Vivas, M. y Saltos-Dueñas, C. (2017). La importancia del aprendizaje y conocimiento del idioma inglés en la enseñanza superior. Dominio de las Ciencias, 3(3), 759-771. Doi: doi.org/10.23857/dc.v3i3 mon.707

España, C. (2010). El idioma inglés en el currículo universitario: importancia, retos y alcances. Revista Electrónica Educare, 14(2), 63-69. Recuperado de https://www.redalyc.org/articulo.oa?id=1941/194115606005

Ibáñez, R. (2008). Comprensión de textos académicos escritos en inglés: relación entre nivel de logro y variables involucradas. Revista Signos, 41(67), 203-229. Doi: doi.org/10.4067/S0718-09342008000200008

León-Jiménez, F., Menéndez-Núñez, R., Alberto-Casas, B., Echeandia-Diez, M., PoncePaucar, J., Falla-Albujar, F. y Loayza-Enríquez, B. (2018). Nivel de conocimientos en inglés en ingresantes de una universidad de Lambayeque, 2015-I. Revista Experiencia en Medicina, 4(3). Recuperado de http://www.rem.hrlamb.gob.pe/index.php/REM/article/view/228/158

Martínez-Salas, M. (2019). El modelo pedagógico de clase invertida para mejorar el aprendizaje del idioma inglés. Investigación Valdizana, 13(4). Doi: doi.org/10.33554/riv.13.4.486

Oñate, O. (2016). Factores que inciden en el dominio del inglés a nivel universitario. INNOVARE. Revista Electrónica de Educación Superior, 1(1), 66-99. Recuperado de http://innovare.udec.cl/wp-content/uploads/2016/08/0719-7500.2016.4.pdf 

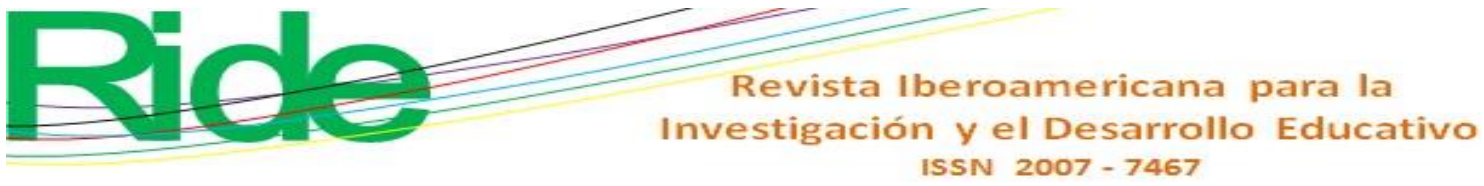

Rueda, M. y Wilburn, M. (2014). Enfoques teóricos para la adquisición de una segunda lengua desde el horizonte de la práctica educativa. Perfiles Educativos, 36(143), 2128.

Recuperado

de

http://www.scielo.org.mx/scielo.php?script=sci_arttext\&pid=S0185$26982014000100018 \& \operatorname{lng}=\mathrm{es} \& \operatorname{tng}=\mathrm{es}$

Soarín, A. (2003). Capítulo 1. El inglés como lengua internacional (tesis doctoral). Recuperado de https://www.tdx.cat/bitstream/handle/10803/10435/cap1.pdf

Tapia, J. (2018). Factores que inciden en el proceso de aprendizaje del inglés como lengua extranjera. Revista Científica Ciencia y Tecnología, 18(18). Doi: doi.org/10.47189/rcct.v18i18.165

Universidad Autónoma del Carmen [Unacar] (2017). Actualización de los fundamentos de la evaluación diagnostica del dominio del idioma inglés (402).

Uribe, J. (2012). Importancia del idioma inglés en las instituciones de educación superior: el caso de la Corporación Universitaria de Sabaneta. Uni-pluri/versidad, 12(2), 97-103. Recuperado d https://revistas.udea.edu.co/index.php/unip/article/view/14441/12676 


\begin{tabular}{|l|l|}
\hline Rol de Contribución & $\begin{array}{c}\text { Revista Iberoamericana para la } \\
\text { Investigac } 2007-7467 \\
\text { ISsn esarrollo Educativo }\end{array}$ \\
\hline Conceptualización & Autor (es) \\
\hline Metodología & Ángel Esteban Torres-Zapata \\
\hline Software & Ángel Esteban Torres-Zapata \\
\hline Validación & José Rafael Villanueva Echavarría \\
\hline Análisis Formal & Teresa del Jesús Brito-Cruz \\
\hline Investigación & Ángel Esteban Torres-Zapata \\
\hline Recursos & Ángel Esteban Torres-Zapata \\
\hline Curación de datos & José Rafael Villanueva-Echavarría \\
\hline $\begin{array}{l}\text { Escritura - Preparación del } \\
\text { borrador original }\end{array}$ & Teresa del Jesús Brito-Cruz \\
\hline $\begin{array}{l}\text { Escritura - Revisión y } \\
\text { edición }\end{array}$ & Teresa del Jesteban Torres-Zapata Brito-Cruz \\
\hline Visualización & José Rafael Villanueva-Echavarría \\
\hline Supervisión & Ángel Esteban Torres-Zapata \\
\hline Administración de Proyectos & Ángel Esteban Torres-Zapata \\
\hline Adquisición de fondos & José Rafael Villanueva-Echavarría \\
\hline
\end{tabular}

